\title{
Correction to: Nivolumab treatment beyond progressive disease in advanced non-small cell lung cancer
}

Takatoshi Enomoto ${ }^{1}$ (D) Akihiro Tamiya ${ }^{1} \cdot$ Kinnosuke Matsumoto $^{1} \cdot$ Yuichi Adachi $^{1} \cdot$ Koji Azuma $^{1} \cdot$ Yuji Inagaki $^{1}$. Shunichi Kouno ${ }^{1} \cdot$ Yoshihiko Taniguchi $^{1} \cdot$ Nobuhiko Saijo $^{1} \cdot$ Kyoichi Okishio $^{2} \cdot$ Shinji Atagi $^{2}$

Published online: 22 February 2022

(c) The Author(s), under exclusive licence to Federación de Sociedades Españolas de Oncología (FESEO) 2022

\section{Correction to:}

Clinical and Translational Oncology (2021) 23:582-590

https://doi.org/10.1007/s12094-020-02452-1

In the original version of this article, the given names of all authors were omitted. The complete names were provided below.

Takatoshi Enomoto, Akihiro Tamiya, Kinnosuke Matsumoto, Yuichi Adachi, Koji Azuma, Yuji Inagaki, Shunichi Kouno, Yoshihiko Taniguchi, Nobuhiko Saijo, Kyoichi Okishio, Shinji Atagi

The original article has been corrected.

The original article can be found online at https://doi.org/10.1007/ s12094-020-02452-1.

\section{Takatoshi Enomoto}

toship0830@gmail.com

1 Department of Internal Medicine, National Hospital Organization Kinki-Chuo Chest Medical Center, 1180 Nagasone-cho, Kita-ku, Sakai, Osaka 591-8555, Japan

2 Clinical Research Center, National Hospital Organization Kinki-Chuo Chest Medical Center, 1180 Nagasone-cho, Kita-ku, Sakai, Osaka 591-8555, Japan
Publisher's Note Springer Nature remains neutral with regard to jurisdictional claims in published maps and institutional affiliations. 